\title{
Shape Optimization of A 3D Slider with Dimples
}

\author{
Michel Cervantes ${ }^{1}$, Samule Cupillard ${ }^{2}$, Kevin Bance ${ }^{3}$ and Michael Kokkolaras ${ }^{4}$ \\ 1. Division of Fluid and Experimental Mechanics, Lulea University of Technology, Lulea 971 87, Sweden \\ 2. Hydro-Québec, Research Institute, Québec J3X 1S1, Canada \\ 3. National Institute for Applied Science, Rouen 76800, France \\ 4. Department of Mechanical Engineering, McGill University, Montreal, Quebec H3A 0C3, Canada
}

\begin{abstract}
Sliding contacts in laminar flow regimes have been investigated extensively in recent years. The results indicate the possibility to increase load carrying capacity in a slider bearing for more than $10 \%$ with the addition of dimples. Parametric studies have been performed to determine optimal size and position, with emphasis in the optimal shape and position of the dimple for an operating condition. In this article, the numerical analysis of a 2D textured slider bearing with a dimple is initially considered with an isothermal laminar fluid. Position, depth, width and convergence ratio are optimized, the results demonstrate the importance of the width and convergence ratio to increase load. Then, the numerical analysis of a 3D textured slider bearing with fore-region and extended channels at the outlet and on the sides of a pad is considered. The simulations are also carried out for a laminar isothermal flow. Three dimples are considered and their depth is optimized.
\end{abstract}

Key words: Sliding contact, laminar, texture, dimple, optimization.

\section{Introduction}

Hydrodynamic contacts are used to support radial and axial forces of rotating shafts. Load carrying capacity and friction force are two characteristics that determine hydrodynamic contacts performance. For economic and safety concerns, many studies have focused on improving the performance of such bearing contacts.

It has been shown that introducing a texture onto a hydrodynamic surface provides benefits as it can increase load carrying capacity and/or reduce friction force [1-3]. A lot of work has been devoted to improving performance of textured bearings. It was shown that texture dimensions and distribution into the contact influence bearing performance [4-6]. Thus, most of the work has been performed by varying texture parameters and studying their effect. This is time consuming and certainly not the most efficient way. The best performance value obtained might not be

\footnotetext{
Corresponding author: Michel Cervantes, professor, research field: fluid mechanics. E-mail:
} michel.cervantes@ltu.se. the optimal one. The question of finding optimal parameter values that yield best performance in a practical manner is thus of great interest. As different parameters of the texture must be optimized simultaneously for different operating conditions, one must develop efficient methods to attain optimality. For hydrodynamic bearings, design variables define the texture geometry (depth, width and density) while the objective function to be optimized is the load carrying capacity and/or friction force.

Rayleigh [7] first optimized an infinitely wide slider bearing. He found the shape that maximized load carrying capacity given the minimum film thickness. Gradient-based algorithms were used to determine optimal design parameter values. Fixing the minimum film thickness, Boedo and Eshkabilov [8] optimized the sleeve geometry of a journal bearing to give the maximum load carrying capacity. The authors used genetic algorithms with Reynolds equation to solve this problem. The method gives results comparable with other published optimization strategies and attains only a small improvement in load carrying capacity. Papadopoulos et al. [9] could optimize the load 
carrying capacity of a 2-dimensional thrust bearing with partial texturing by coupling a CFD (computational fluid dynamics) code with an optimization tool also based on genetic algorithms and local search techniques. Substantial increase in load carrying capacity could be achieved. Gusek et al. [10] used a unified approach to optimize texture shapes of parallel slider bearings. The approach works for bearings with fluid flow governed by the Reynolds equation. For a hydrodynamic slider bearing partially textured, Rahmani et al. [11] used an optimization procedure to get maximum load carrying capacity and minimum friction coefficient using the Reynolds equation. A genetic algorithm was used to obtain optimal geometric parameter values.

In this paper, the optimization of a slider bearing is considered. A 2-dimensional model is initially investigated with one dimple. Then a 3-dimensional slider with three dimples, fore-region and extended channels at the outlet and on the sides of a pad is investigated.

\section{Numerical Model}

Two-dimensional and three-dimensional models were used to model the sliding contact with an isothermal laminar flow.

\subsection{Equations}

The Navier-Stokes equations and continuity for an isothermal fluid were solved over the domains considered.

$$
\begin{aligned}
& \frac{\partial U}{\partial t}+U \cdot \nabla U=\frac{1}{\rho} \nabla P+\frac{\mu}{\rho} \nabla^{2} U \\
& \nabla \cdot U=0
\end{aligned}
$$

The finite volume method together with second order schemes were used to discretize and solve the equations. The commercial code CFX 13.0 was used to performed the simulation

\subsection{Geometry and Boundary Conditions}

The 2D geometry investigated is presented in Fig. 1.
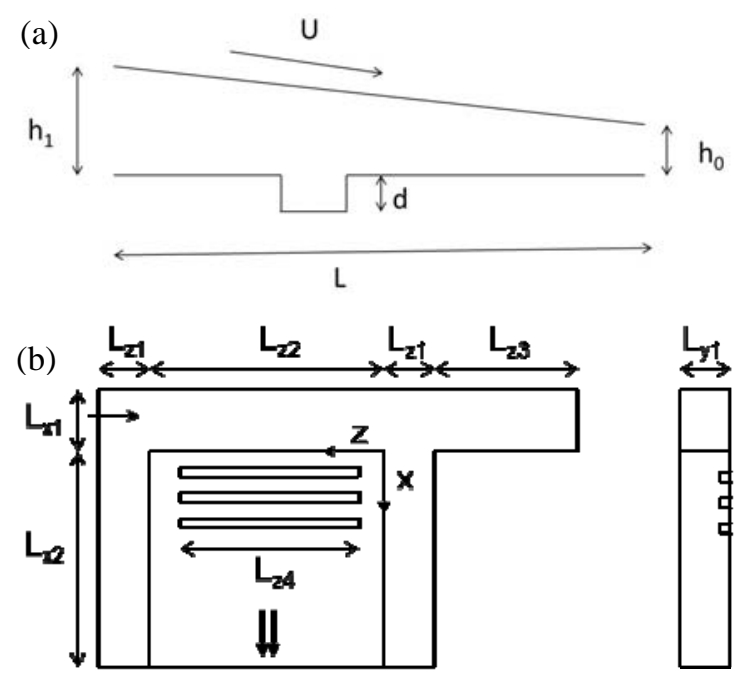

Fig. 1 Investigated geometry: (a) two-dimensional; (b) three-dimensional.

A similar configuration was previously investigated by Cupillard et al. [12]. The dimensions are: length $\mathrm{L}=6$ $\mathrm{mm}$, initial inlet height $\mathrm{h}_{01}=0.045 \mathrm{~mm}$, outlet height $\mathrm{h}_{0}$ $=0.03 \mathrm{~mm}$, initial dimple depth $\mathrm{d}_{0}=0.015 \mathrm{~mm}$ and initial dimple width $\mathrm{w}_{0}=0.3 \mathrm{~mm}$. The initial inlet height corresponds to a convergence ratio $\mathrm{k}=$ $\left(h_{01}-h_{0}\right) / h_{0}=0.5$. The effects of dimples on the load carrying capacity are significant with such a convergence ratio according to Cupillard et al. [12]. The dimple leading edge is initially situated at $l_{0}=0.2$ $\mathrm{mm}$ from the slider inlet.

For all cases, the sliding velocity is $\mathrm{U}=30 \mathrm{~m} / \mathrm{s}$ and the kinematic viscosity of the fluid is $v=1.27 \mathrm{e}^{-5} \mathrm{~m}^{2} / \mathrm{s}$. The equivalent Reynolds number based on the minimum film thickness is $\mathrm{Re}=71$. The inlet of the slider is considered as opening, i.e., flow may enter or leave the inlet. The outlet allows only flow out of the domain. All walls are assumed smooth.

The 3D geometry investigated is presented in Fig. 1, right schematic. The geometry has previously been investigated by Cupillard et al. [12] under laminar regimes and Cervantes et al. [13] under a turbulent regime. The dimensions of the $3 \mathrm{D}$ slider bearing are presented in Table 1 . The first dimple is located $\mathrm{l}_{0}=0.2$ $\mathrm{mm}$ from the pad inlet and the distance between the dimples is set to $0.4 \mathrm{~mm}$. The dimples width is $\mathrm{w}_{0}=0.3$ $\mathrm{mm}$. The sliding velocity is $\mathrm{U}=30 \mathrm{~m} / \mathrm{s}$ and the 
Table 1 Dimensions in $\mathrm{mm}$ of the slider bearing (Fig. 1) (right schematic).

\begin{tabular}{lllllllll}
\hline $\mathrm{L}_{\mathrm{x} 1}$ & $\mathrm{~L}=\mathrm{L}_{\mathrm{x} 2}$ & $\mathrm{~L}_{\mathrm{x} 3}$ & $\mathrm{~L}_{\mathrm{y} 1}$ & $\mathrm{~L}_{\mathrm{y} 2}$ & $\mathrm{~L}_{\mathrm{z} 1}$ & $\mathrm{~L}_{\mathrm{z} 2}$ & $\mathrm{~L}_{\mathrm{z} 3}$ & $\mathrm{~L}_{\mathrm{z} 4}$ \\
\hline 1.68 & 6 & 0.3 & 0.96 & 0.0225 & 1 & 6 & 4 & 5 \\
\hline
\end{tabular}

Lx3: dimple width, Ly2: dimple depth

kinematic viscosity of the fluid is $v=1.27 \mathrm{e}^{-5} \mathrm{~m}^{2} / \mathrm{s}$, similarly to the $2 \mathrm{D}$ dimple. The convergence ration was set to 1 with a minimum film thickness $h_{0}=30 \mu \mathrm{m}$. The equivalent Reynolds number based on the minimum film thickness is $\operatorname{Re}=71$. The inlet mass flow was set to $\mathrm{Q}=50 \mu \mathrm{kg} / \mathrm{s}$.

The following geometric parameters are considered as optimization variables:

- Dimple depth $d$;

- Dimple width w;

- Dimple position l;

- Entrance height $h_{1}$.

In the presence of multiple dimples, the subscript $i$ is used to denote dimple, e.g., $d_{2}$ denotes the depth of the second dimple, where the first dimple is closest to the entrance.

\subsection{Discretization and Convergence}

Hexahedral meshes were used for all simulations. The meshes were created with a variable number of elements function of the value of the parameter(s) investigated.

For the 2D slider, the entrance had 21 nodes and about 1,200 cells were used in the bearing length, where a node was placed every $5 \mu \mathrm{m}$. The dimple had two nodes per micrometer in the vertical direction with a minimum of 6 nodes. Such node distribution means a mesh with about 24,000 hexahedral cells, which gives a load error of about $0.3 \%$ due to space discretization (Cupillard et al. [12]). All simulations were run until a maximum residual of $10^{-9}$ was reached.

The 3D slider had a total of about 300,000 hexahedral cells. Similarly to the 2D case, the mesh was adjusted function of the geometry. In the present work, the dimple depth was varied and the mesh adjusted as for the 2D case. All simulations were run until a maximum residual of $10^{-5}$ was reached. The residual is a compromise between numerical accuracy and simulation time.

\subsection{Optimization}

The flow analysis solvers were integrated with an optimization approach implemented in the Matlab computational environment. To increase the likelihood of finding a global optimum, Matlab's Global Optimization Toolbox was used. Specifically, a multi-start strategy that first uses a scatter-search algorithm to sample the design space and generate a number of points with promising objective and constraint values was used [14]. Then, a number of general nonlinear optimizations using Matlab's implementation of the SQP (sequential quadratic programming) algorithm (Matlab function "fmincon") with these points as initial guesses were executed. Finally, the obtained solutions to determine which solution may be a possible global optimum were analyzed. Fig. 2 depicts the flow diagram of the analysis/optimization loop and summarizes the multi-start optimization strategy of the Global Search method of the Matlab Global Optimization toolbox.

The mathematical formulation of the optimization problems is:

- Maximize the load: $f(x)=\iint_{S} P d y d z$, where $S$ is the upper surface of the bearing;

- Vector of optimization variables: $x=\left[d, w, l, h_{1}\right]$;

- General constraints: $g(x)=\left(\sum\left(1_{i}+w_{i}\right)-L\right) \leqq 0$;

- Simple variable bound constraints: $l_{b} \leqq x \leqq u_{b}$, where $\mathrm{l}_{\mathrm{b}}$ and $\mathrm{u}_{\mathrm{b}}$ are lower and upper bound vectors, respectively. The lower and upper bounds were chosen as follow for the different variables: $1 \leqq d \leqq$ $50,1 \leqq w \leqq 5,900,1 \leqq l \leqq 5,900,30 \leqq h_{1} \leqq 100$, values in $\mu \mathrm{m}$.

The initial vector $x_{0}$ to initiate the optimization was determined from optimization of single variables using the function fminbnd. The function allows finding local maximum in a one dimension bounded interval. This function is appropriate since the 

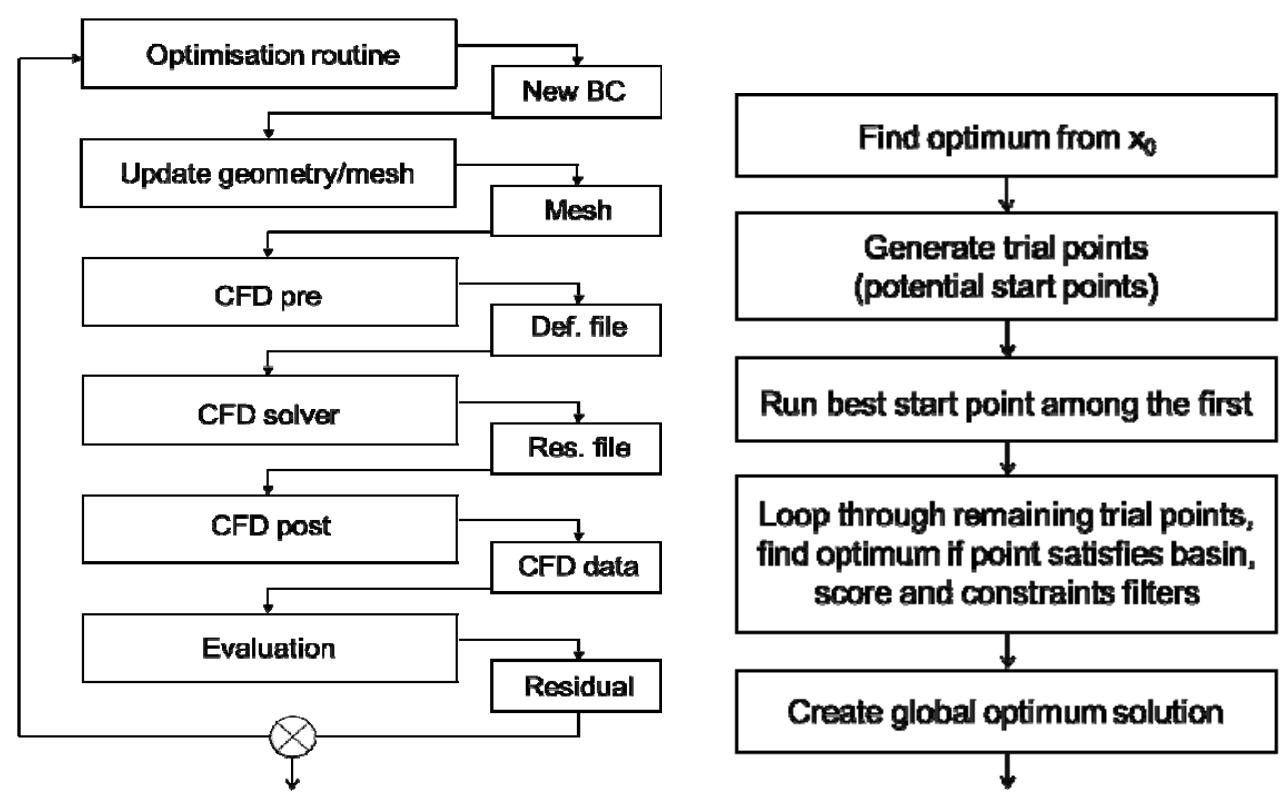

Fig. 2 Analysis/optimization loop and strategy.

behavior of each variable is well known, i.e., the load has a single maximum in the interval investigated [12].

\section{Results}

\subsection{Geometry}

A single parameter optimization was initially performed to confirm previous finding and obtain an initial guess. The results are presented in Table 2 together with the results of a smooth geometry and the reference geometry with one dimple $\left(d_{0}=15 \mu \mathrm{m}, w_{0}=\right.$ $\left.300 \mu \mathrm{m}, l_{0}=200 \mu \mathrm{m}, \mathrm{h}_{01}=45 \mu \mathrm{m}\right)$. Contour plots of the axial velocity are presented in Fig. 3.

The introduction of one dimple increases the load with $1.7 \%$ compared to the smooth case, see reference geometry with one dimple in Table 2. The optimal depth of the dimple is found to be $d=9 \mu \mathrm{m}$. The optimum is obtained when recirculation in the dimple is minimized, similarly to Cupillard et al. [12]. The influence of the dimple width is found to have the largest positive effect on the load: $+46 \%$ for $w=4,049$ $\mu \mathrm{m}$. The width of the dimple is about $2 / 3$ of the bearing length. This result converges in fact to the step bearing found by Rayleigh [7]. Separation in the dimple is also moderate and localized near the dimple leading edge. Similarly to the depth, the dimple position has a minor influence on the results. For $l=1,775 \mu \mathrm{m}$, the load increases with $2.7 \%$. Of interest is the large amount of recirculation found in the dimple, indicating that more load can be gained by minimizing the depth at this position. The entrance is found to have a large impact on the load carrying capacity. For $\mathrm{h}_{01}=70 \mu \mathrm{m}$ or $\mathrm{a}$

Table 2 Single parameter optimization (*number of iteration to obtained the optimum).

\begin{tabular}{|c|c|c|c|c|c|c|}
\hline & Reference smooth & Reference dimple & Opti. 1.1 (d) & Opti. 1.2 (w) & Opti. 1.3 (l) & Opti. $1.4\left(\mathrm{~h}_{1}\right)$ \\
\hline Length (m) & & & 9 & 4,049 & 1,775 & 70 \\
\hline Load $\times 1 \mathrm{e}^{-5}(\mathrm{~N})$ & 1,571 & 1,598 & 1,605 & 2,293 & 1,613 & 2,115 \\
\hline Variation (\%) & 0 & 1.7 & 2.2 & 46 & 2.7 & 34.6 \\
\hline Number iter. opt. & - & - & 15 & 23 & 32 & 22 \\
\hline
\end{tabular}




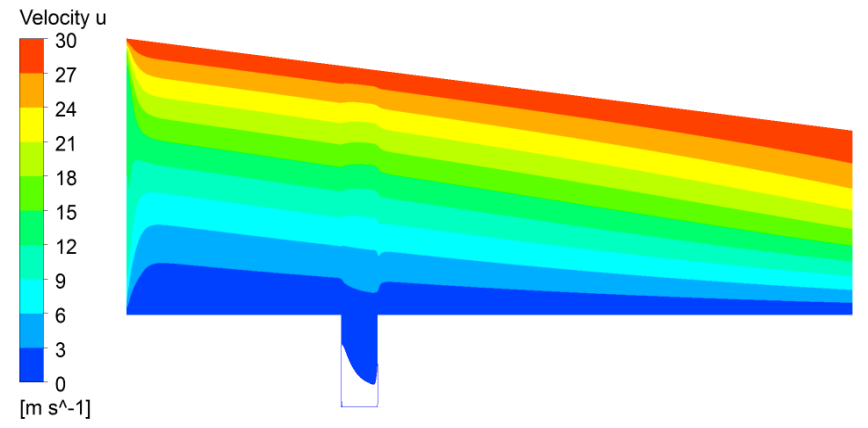

(a) Optimal dimple position.

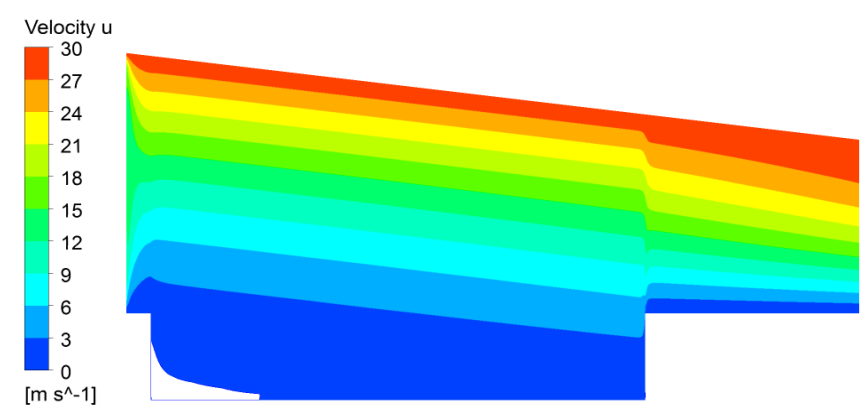

(c) Optimal dimple width.

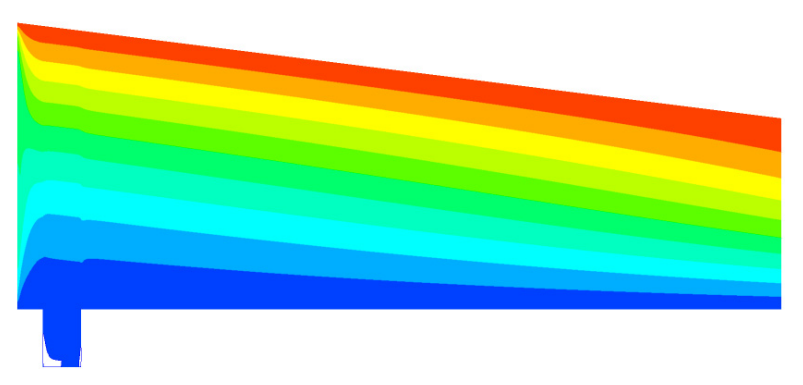

(b) Optimal dimple depth.

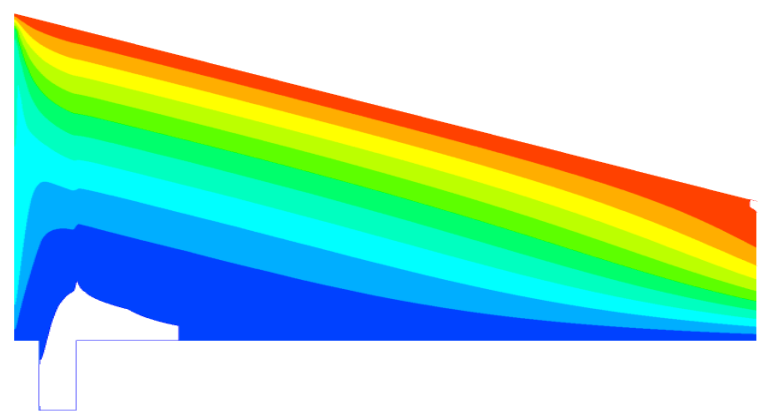

(d) Optimal entrance height.

Fig. 3 Contour plot of the axial velocity for the different optimums, a 1/10 scaling is used in the sliding-direction.

convergence ratio $\mathrm{k}=1.33$, the load increases with $34.6 \%$. A large separation region globing the dimple is found indicating that this dimple depth is not optimal. The number of iterations is small for all cases, between 15 and 32 .

Development of the pressure along the bearing on the sliding wall is presented in Fig. 4 for the different cases investigated. In all cases the pressure increases up to about $2 / 3$ of the bearing length and thereafter decreases, illustrating the bearing functioning. Losses should be minimized in the region of positive pressure gradient to transfer as much energy as possible to the fluid by shear in order to build up pressure. However, losses should be enhanced in the region of negative pressure gradient in order to decrease the pressure to the reference pressure. The optimum appears for attached flow in the first region, i.e., minimum velocity allowing minimum losses. The negative pressure at the bearing inlet is localized to the first cell and is due to the large wall velocity $\mathrm{U}=30 \mathrm{~m} / \mathrm{s}$.

Optimization studies with respect to several parameters were conducted next. The optimal values

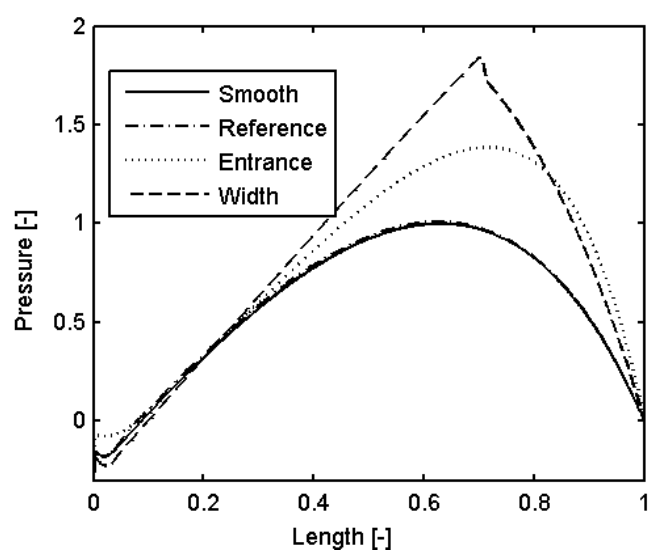

Fig. 4 Pressure variation along the slider for different optimum, the results obtained for optimized depth and position are on the graph but without legend since similar to the smooth and reference cases.

from Table 2 were used as initial guess. The results are reported in Table 3. None of the optimization runs was able to reach the highest load obtained in single-variable optimization, except Opti. 4.2 in Table 3 The reasons are attributed to the initial guess and the noisy character of the objective function. Opti. 4.2 was started from the results of Opti. 1.2. A significant increase in load was obtained: $+69.3 \%$. The results 
complete the results obtained with a single parameter optimization. Entrance height and dimple width are the parameters giving the largest load improvement. However, both of them cannot simultaneously contribute to a significant load improvement as they influence the fluid in the same way: their optimum is obtained before separation. However, the entrance height seems to have a leading effect to increase load carrying capacity.

\subsection{D Geometry}

The flow in the 3D geometry with fore-region and extended channels at the outlet and on the sides is complex (Fig. 5). The fluid entering the numerical domain near the upper wall is entrained in the nearest channel due to the large upper-wall velocity, while the rest of the fluid enters the fore region. Some fluid in the channel recirculates back to the inlet and the rest of the fluid goes down the channel, i.e., some recirculation is present in the channel. The fluid in the fore region is a combination of fluid from the inlet, previous pad and first channel. A large recirculation zone develops due to the large upper wall velocity. Some of the fluid in the fore-region enters the pad, while the rest of the fluid is directed to the second channel or the outlet.

The initial guess to perform the optimization of the three dimples depth was extrapolated from the 2D optimization. The depth of the first dimple was set to $d_{2}$ $=9 \mu \mathrm{m}$, the optimal value obtained in the single-variable optimization study. The depth of the second and third dimples was calculated to have the same distance between the sliding wall and the bottom as the first dimple in order to look like a step bearing. The values $d_{2}=12.5 \mu \mathrm{m}$ and $d_{3}=16 \mu \mathrm{m}$ were chosen.

Table 3 Results of multi-variate optimization studies.

\begin{tabular}{|c|c|c|c|c|c|c|c|c|}
\hline & $\begin{array}{l}\text { Ref. } \\
\text { smooth }\end{array}$ & $\begin{array}{l}\text { Ref } \\
\text { dimple }\end{array}$ & $\begin{array}{l}\text { Opti. } 2.1 \\
\left(\mathrm{w}, \mathrm{h}_{1}\right)\end{array}$ & $\begin{array}{l}\text { Opti. } 2.2 \\
\left(1, h_{1}\right)\end{array}$ & $\begin{array}{l}\text { Opti. } 3.1 \\
\left(\mathrm{~d}, \mathrm{w}, \mathrm{h}_{1}\right)\end{array}$ & $\begin{array}{l}\text { Opti.3.2 } \\
\left(\mathrm{w}, \mathrm{l}, \mathrm{h}_{1}\right)\end{array}$ & $\begin{array}{l}\text { Opti. } 4.1 \\
\left(\mathrm{~d}, \mathrm{w}, \mathrm{l}, \mathrm{h}_{1}\right)\end{array}$ & $\begin{array}{l}\text { Opti. } 4.2 \\
\left(\mathrm{~d}, \mathrm{w}, \mathrm{l}, \mathrm{h}_{1}\right)\end{array}$ \\
\hline Depth (m) & 0 & 15 & 15 & 15 & 17 & 15 & 7.9 & 8.7 \\
\hline Width (m) & 0 & 300 & 3,167 & 300 & 453 & 1,581 & 1,555 & 34 \\
\hline Position (m) & 0 & 200 & 200 & 3,167 & 200 & 2,798 & 1,527 & 19.7 \\
\hline Entrance (m) & 45 & 45 & 64.5 & 64.5 & 47.2 & 69.6 & 63.4 & 72.2 \\
\hline Load $\times 1 e^{-5}(\mathrm{~N})$ & 1,571 & 1,598 & 2,102 & 2,126 & 2,270 & 2,205 & 2,169 & 2,659 \\
\hline Variation (\%) & 0 & 1.7 & 33.8 & 35.3 & 44.5 & 40.3 & 38.1 & 69.3 \\
\hline Number iteration & - & - & 287 & 284 & 270 & 267 & 354 & 218 \\
\hline
\end{tabular}
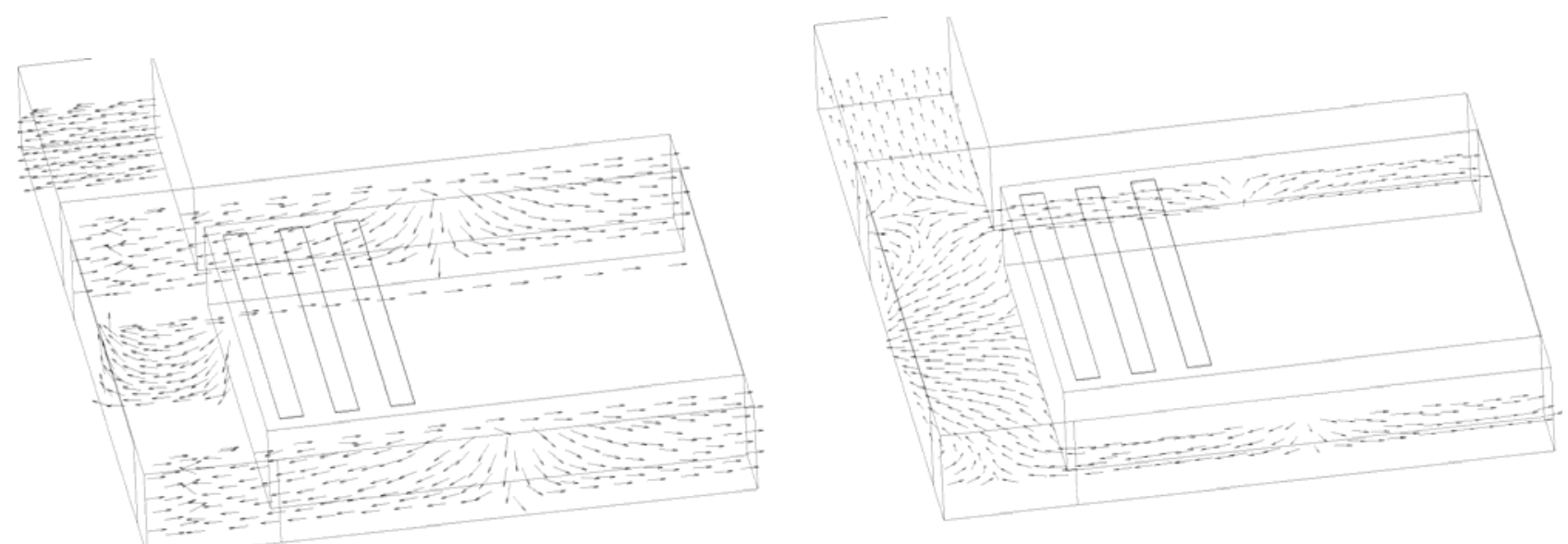

Fig. 5 Normalized velocity vector in the fore-region and side channels to illustrate vortex formation and recirculation region. 
The result of the optimization is presented in Table 4 together with the results of the smooth case. An increase of $2.7 \%$ of the load is achieved. The number of iteration is not as important as for the $2 \mathrm{D}$ case due to the larger amount of computer capacity needed to achieve each simulation. The result is opposite to the one expected: the depth of the first dimple is the largest and the depth of the following dimples decreases along the pad (Fig. 6). The contour plot of the axial velocity points out some recirculation in the first and second dimple. This is confirmed by contour plots of the wall shear stress on the pad in the sliding direction. The separation region is symmetrical to the pad mid-plane but varies in each dimple. The flow is more attached near the channels than in the middle of the pad. As a matter of fact, there is some side leakage, i.e., a transverse velocity component in Fig. 7. The side leakage decreases the occurrence of separation. The variation of the separation region indicates the necessity of a variable depth for each dimple: deeper dimples close to the channel and shallower near the pad middle.

The optimal dimples depth is quite different than expected. A step like bearing was expected, the pressure variation along the slider in the middle of the pad is presented in Fig. 8 for the smooth case together with the result of the geometry with dimples. The pressure difference between both geometries is also presented. At the entrance of the pad, a sudden increase of the pressure occurs, the effect is known as the ram pressure effect [15]. A small pressure decrease occurs immediately after the ram pressure effect. Then, the pressure increases up to $2 / 3$ of the pad length to decrease to the fore-region pressure, which is nearly zero in the present case. The introduction of the three dimples creates a wavy development of the pressure in the region of positive pressure gradient. The pressure is initially lower with dimples than for the smooth case, up to the middle of the second dimple. Then, the pressure is larger with the presence of dimples. The decreasing dimple depth may be explained by the fluid mean velocity. The distance between the upper- and lower-wall decreases along the pad. The lower area involves higher mean velocity (side leakage is neglected in the reasoning), which promotes separation in deeper dimples. Therefore, the dimples depth should decrease along the pad to minimize separation and increase load.

Table 4 Dimples depth optimization for the 3D slider.

\begin{tabular}{lll}
\hline & $\begin{array}{l}\text { Reference } \\
\text { smooth }\end{array}$ & $\begin{array}{l}\text { Opti. 4.1 } \\
\left(\mathrm{d}_{1}, \mathrm{~d}_{2}, \mathrm{~d}_{3}\right)\end{array}$ \\
\hline Depth 1, 2, 3 (m) & $0,0,0$ & $17.5,13.8,11,9$ \\
Width (m) for i=1,2,3 & 300 & 300 \\
Position (m) & $200,900,1,600$ & $200,900,1,600$ \\
Entrance (m) & 60 & 60 \\
Load (N) & 7.64 & 7.84 \\
Variation (\%) & 0 & 2.7 \\
Number iteration & - & 117 \\
\hline
\end{tabular}
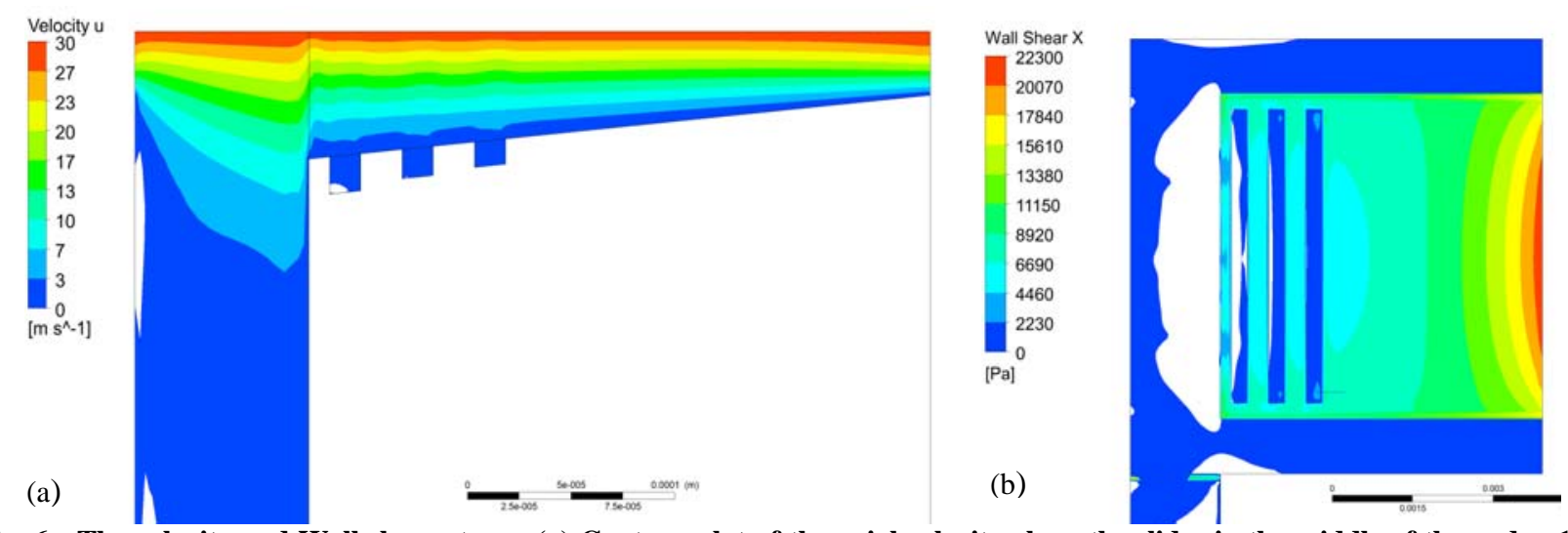

Fig. 6 The velocity and Wall shear stress: (a) Contour plot of the axial velocity along the slider in the middle of the pad, a 1/10 scaling is used in the sliding-direction; (b) Wall shear stress in the sliding direction, negative values indicating a recirculation are clipped. 


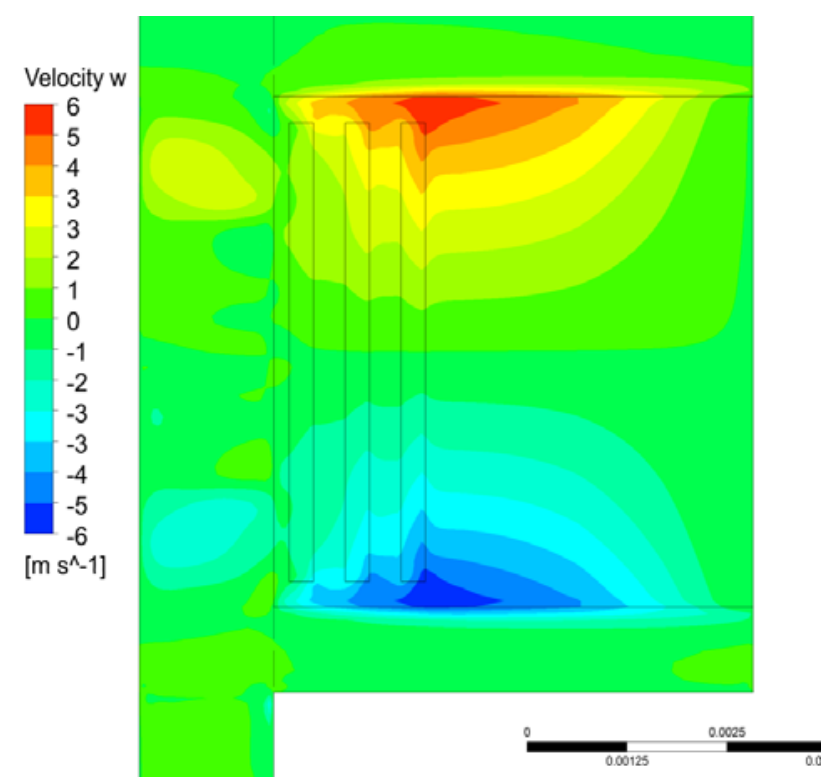

Fig. 7 Contour plot of the velocity orthogonal to the siding velocity: transverse velocity illustrating side leakage in 3D sliding bearing with side channels.
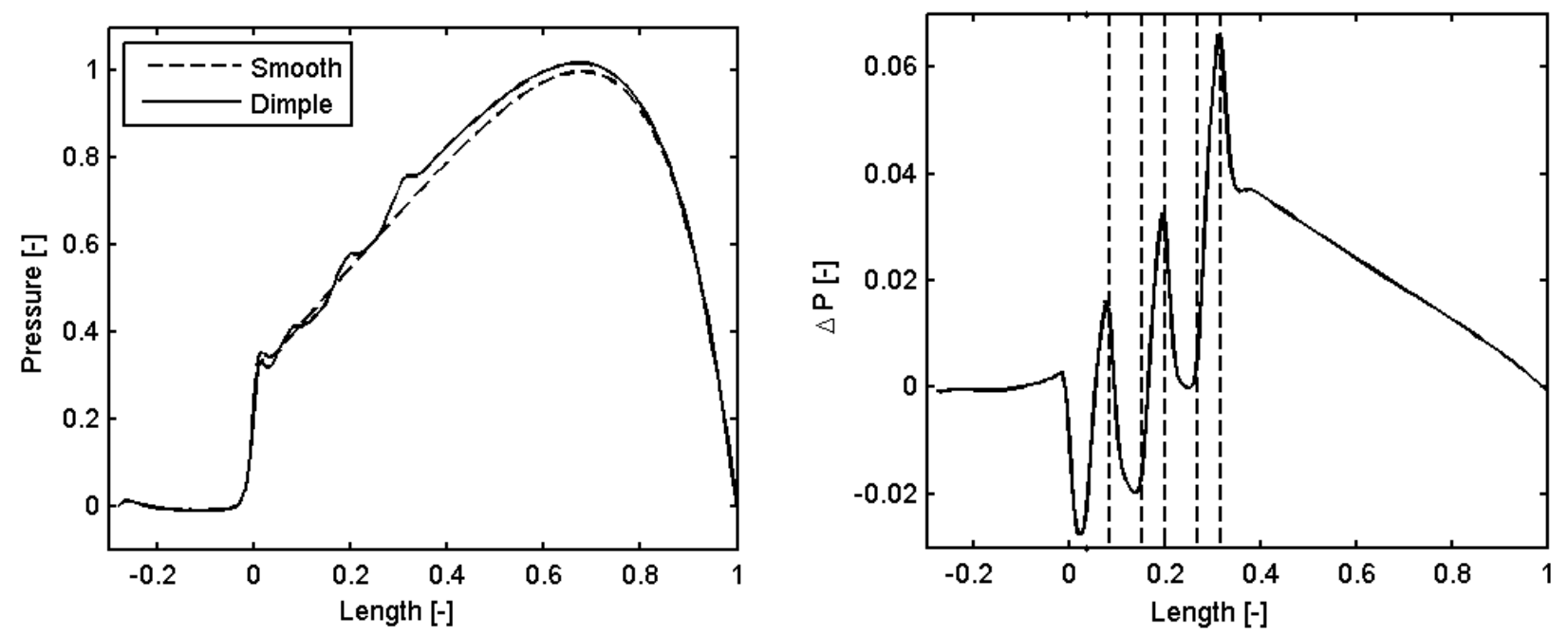

Fig. 8 Pressure variation along the slider in the middle of the pad (right) and pressure difference between the dimple geometry and the smooth geometry, the results are normalized with the maximum pressure obtained with the smooth geometry, the dash lines represent the position of the dimples.

\section{Conclusions}

The numerical optimization of a 2D slider bearing with a dimple was considered with an isothermal laminar fluid. Position, depth, width and convergence ratio were optimized; the results demonstrate the importance of the width and convergence ratio to increase load. Multi-variate optimization studies highlight the importance of the initial guess for finding a global optimum. With an appropriate initial guess, the load was improved by as much as $69 \%$ relative to the smooth case.

The numerical analysis of a 3D textured slider bearing with fore-region and extended channels at the outlet and on the sides of a pad was also considered. The simulations were carried out for a laminar isothermal flow. Three dimples were considered and their depth was optimized. The results show that the dimple depth should decrease along the pad in the sliding direction. This is opposite to using a step 
bearing, and may be attributed to the increased velocity along the pad, which necessitates shallower dimples to avoid separation. Furthermore, the presence of side leakage decreases the occurrence of separation near the channels, indicating the necessity of a variable depth in each dimple: deeper near the channels and shallower in the middle of the pad.

\section{Acknowledgments}

The research presented was carried out as a part of the "Swedish Hydropower Center-SVC". SVC has been established by the Swedish Energy Agency, Elforsk and Svenska Kraftnät together with Lulea University of Technology, The Royal Institute of Technology, Chalmers University of Technology and Uppsala University (www.svc.nu).

\section{References}

[1] I. Etsion, Improving tribological performance of mechanical components by laser surface texturing, Tribology letters 17 (4) (2004) 733-737.

[2] N. Tala-Ighil, P. Maspeyrot, M. Fillon and A. Bounif, Effects of surface texture on journal bearing characteristics under steady state operating conditions, in: Proceedings of the Institution of Mechanical Engineering Tribology 221 (6) (2007) 623-633.

[3] K. Tonder, Inlet roughness tribodevices: Dynamic coefficients and leakage, Tribology International 34 (12) (2001) 847-852.

[4] R. B. Siripuram and L. S. Stephens, Effect of deterministic asperity geometry on hydrodynamic lubrication, Journal of Tribology 126 (3) (2004) 527-534.

[5] T. H. Yu and F. Sadeghi, Groove effects on thrust washer lubrication, Journal of Tribology 123 (2) (2001) 295-304.
[6] M. B. Dobrica, M. Fillon and M. D. Pascovici, T. Cicone, Texturing effects in plane-inclined slider bearings, in: STLE/ASME International Joint Tribology Conference, San Diego, California, USA, 2007.

[7] L. Rayleigh, Notes on the theory of lubrication, Philosophical Magazine and Journal of Science 35 (1918) $1-12$.

[8] S. Boedo and S. L. Eshkabilov, Optimal shape design of steadily loaded journal bearings using genetic algorithms, Tribology Transactions 46 (1) (2003) 134-143.

[9] C. I. Papadopoulos, P. G. Nikolakopoulos and L. Kaiktsis, Evolutionary optimization of micro-thrust bearings with periodic partial trapezoidal surface texturing, Journal of Engineering for Gas Turbines and Power 133 (2011) $1-10$.

[10] A. Guzek, P. Podsiadlo and G. W. Stachowiak, A unified computational approach to the optimization of surface textures: One dimensional hydrodynamic bearings, Tribology Online, Japanese Society of Tribologists 5 (3) (2010) 150-160.

[11] R. Rahmani, I. Mirzaee, A. Shirvani and H. Shirvani, An analytical approach for analysis and optimisation of slider bearings with infinite width parallel textures, Tribology International 43 (2010) 1551-1565.

[12] Z. Ugray, L. Lasdon, J. Plummer, F. Glover, J. Kelly and R. Marti, Scatter search and local NLP solvers: A multistart framework for global optimization, INFORMS Journal on Computing 19 (3) (2007) 328-340.

[13] S. Cupillard, M. J. Cervantes and S. Glavatskih, Pressure build up mechanism in a textured inlet of a hydrodynamic contact, ASME J. Tribol. 130 (2) (2008) 10.

[14] M. J. Cervantes and S. Cupillard, 3D thermodynamic analysis of a turbulent textured slider, in: Proceeding of the 21st International Symposium on Transport Phenomena, Taiwan, 2010.

[15] J. X. Zhang and C. M. Rodkiewicz, On the design of thrust bearings using a CFD technique, Tribology Transactions 40 (3) (1997) 403-412. 